\title{
Red cell distribution width is a potential predictor of early relapse in polymyalgia rheumatica
}

\author{
D. Soddu',2, D. Sola'-3, M. Bellan'1-3, E. Boin'1, M.G. Cittone ${ }^{1}$, E. Zecca ${ }^{1,2}$, \\ E. Matino ${ }^{1,2}$, R. Pedrazzoli', E. Rizzi ${ }^{1,2}$, A.R. Pedrinelli',, S. Tonello', V. Vassia ${ }^{1,2}$, \\ R. Landi ${ }^{1,2}$, M. Pirisi ${ }^{1-3}$, P.P. Sainaghi ${ }^{1-3}$ \\ ${ }^{1}$ Department of Translational Medicine, University of Piemonte Orientale UPO, Novara; ${ }^{2}$ Division of Internal \\ Medicine, Immunorheumatology Unit, "Maggiore della Carità" Hospital, Novara; ${ }^{3} C A A D$, Centre for \\ Autoimmune and Allergic Diseases, Novara, Italy
}

\begin{abstract}
SUMMARY
Red blood cell distribution width (RDW) has been studied as a prognostic biomarker for different chronic inflammatory diseases. In this paper we aim to evaluate its potential role in the prediction of early relapse in patients affected by polymyalgia rheumatica (PMR).

We revised retrospectively clinical records of patients who received a diagnosis of PMR, according to 2012 ACR/EULAR classification criteria, for whom baseline clinical and laboratory data were available. The baseline RDW variation coefficient was correlated to the risk of relapse, in the first 6 months of the disease.

We identified 44 patients [females 15 (34.0\%)/males 29 (66.0\%); median age 80 (72-83)], 9 of whom had an early relapse. These patients showed a larger median RDW than patients who did not relapse [13.7 (13.5$14.9) \%$ vs 13.5 (12.7-14.2)\%; $\mathrm{p}=0.04)$. The two groups were comparable for all the other clinical and laboratory parameters considered. Interestingly, patients in the higher half of the RDW distribution showed a shorter relapse-free survival $(\mathrm{p}<0.03)$. In a stepwise logistic regression, $\mathrm{RDW}(\mathrm{p}=0.01)$ predicted the risk of relapse at 6 months, while age, gender, CRP, ESR, Hb, MCV and prednisone dose did not fit the model.

Our results show that RDW is an independent biomarker of early relapse, making this parameter a potentially promising predictive marker in PMR.
\end{abstract}

Key words: Red cell distribution width, polymyalgia rheumatica, relapse, biomarker.

\section{INTRODUCTION}

ed cell distribution width (RDW), routinely reported as a component of the complete blood count, evaluates the variability in size of circulating erythrocytes, thus being potentially useful in the differential diagnosis of anaemia. Recently, RDW has been successfully tested as a prognostic biomarker in different human diseases, such as sepsis (1), coronary artery disease (2), and venous thromboembolism (3). More generally, RDW seems to predict overall mortality, which has shown to increase by $22 \%$ for every $1 \%$ increase of RDW (4).

Moreover, RDW is associated with disease activity in various rheumatic diseases, such as systemic lupus erythematosus (SLE),
Sjögren's syndrome $(5,6)$ and rheumatoid arthritis (RA) (7). RDW is also increased in systemic sclerosis, in which disease it suggests cardiopulmonary involvement (8). Finally, RDW may predict the response to treatment in SLE and in RA patients $(5,9)$. To the best of our knowledge, the potential prognostic role of RDW in the prediction of response to treatment has never been evaluated in polymyalgia rheumatica (PMR) so far. PMR is a common inflammatory disease of the elderly, which usually responds well to steroids, despite it may be associated with relapses. Currently, there are no clear predictors of PMR relapse, which, on the contrary, might be useful to tailor steroid tapering and, in selected cases, to add disease modifying antirheumatic drugs (DMARD) (10).
Corresponding author: Mattia Bellan

Department of Translational Medicine Università del Piemonte Orientale UPO Via Solaroli, 17 - 28100 Novara (NO), Italy E-mail: bellanmattia@yahoo.it 
In this study, we aimed to explore whether baseline RDW in PMR patients is associated to an early relapse.

\section{MATERIALS AND METHODS}

We performed a retrospective analysis of all the clinical records of patients followedup at our Immuno-Rheumatology clinic between $1^{\text {st }}$ January 2012 and $1^{\text {st }}$ January 2020. The study was conducted in strict accordance with the principles of the Declaration of Helsinki.

We identified all the patients who received a provisional diagnosis of isolated PMR according to the 2012 American College of Rheumatology/European League against Rheumatism (ACR/EULAR) classification Criteria (11). We selected 70 subjects who were tested with a laboratory panel at our central laboratory at baseline. We excluded $18 / 70$ patients, because the provisional diagnosis of PMR was not confirmed during the follow-up. Subsequently, we also excluded from the analysis 8 more patients who, at the time of diagnosis, were older than 90 . This choice was motivated by the observation that RDW increases with age and loses its predictive significance in the oldest old (12).

Therefore, the population selected for the statistical analysis comprised 44 patients. Besides demographics and treatment history (prednisone and/or drug-modifying anti-rheumatic drugs, DMARD) we recorded the following data for each patient at baseline and at relapse:

1) inflammatory markers: erythrocyte sedimentation rate (ESR) and C-reactive protein (CRP);

2) RDW, expressed as variation coefficient;

3) hemoglobin;

4) mean corpuscular volume (MCV) and mean corpuscular hemoglobin (MCH).

Early relapse was evaluated if it appeared within 6 months from the diagnosis, according to both clinical and laboratory criteria, as suggested by ACR/EULAR (13).

\section{Statistical analysis}

All the data were recorded anonymously in a database and analysed by the statistical software package Statistica for Windows, StatSoft Inc. 12. The normal distribution was assessed by Shapiro-Wilk test; following the non-normal distribution of the continuous variables, the measures of centrality and dispersion of data chosen were medians and the interquartile range [IQR]. Continuous variables between relapsers and non-relapsers were compared by Mann-Whitney test.

Comparisons of categorical variables were performed using Pearson's $\chi^{2}$ test. The Kaplan-Meier analysis was performed to evaluate the predictive value of RDW for early relapse and survival curves were compared with the Log-Rank test.

Finally, in order to control potential confounders, we built a multiple regression analysis and a stepwise logistic regression for predictors of early relapse in PMR.

The level of significance chosen for all statistical analysis was 0.05 (two-tailed).

\section{RESULTS}

Table I reports the main features of the study population. In the first 6 months from the clinical diagnosis, 9/44 patients (20.4\%) experienced a relapse. Table II

Table I - Clinical features of the study population.

\begin{tabular}{|l|l|}
\hline Age, years & $80[72-83]$ \\
\hline Gender, F/M & $15(34.0 \%) / 29(66.0 \%)$ \\
\hline RDW, \% & $13.5[12.9-14.3]$ \\
\hline Hb, gr/dl & $12.4[11.9-13.3]$ \\
\hline HCT, \% & $38.5[36.7-40.5]$ \\
\hline CRP, mg/dL & $3.5[1.6-5.9]$ \\
\hline ESR, mm/h & $41[34-52]$ \\
\hline $\begin{array}{l}\text { Prednisone dose, mg/ } \\
\text { day }\end{array}$ & $15.0[15.0-15.0]$ \\
\hline MCV, fL & $88.6[85.9-91.7]$ \\
\hline MCH, pg & $28.6[27.7-29.7]$ \\
\hline
\end{tabular}

Continuous variables are presented as medians [Interquartile range]. Categorical variables are shown as frequencies (\%). Abbreviations: F, females; M, males; RDW, red cell distribution width; $\mathrm{Hb}$, haemoglobin; $\mathrm{HCT}$, haematocrit; $\mathrm{CRP}$, C-reactive protein; ESR, erythrocyte sedimentation rate; MCV, mean corpuscular volume; $\mathrm{MCH}$, mean concentration of haemoglobin. 
Table II - Differences between relapsers and non-relapsers in the first 6 months of disease.

\begin{tabular}{|l|c|c|c|}
\hline & $\begin{array}{c}\text { Relapsers } \\
\mathbf{N}=9(20.4)\end{array}$ & $\begin{array}{c}\text { Non relapsers } \\
\mathrm{N}=35(79.6)\end{array}$ & $\mathbf{p}$ \\
\hline Age, years & $80[67-81]$ & $80[73-83]$ & 0.47 \\
\hline Gender, F/M & $5(55.5) / 4(44.4)$ & $24(68.6) / 11(31.4)$ & 0.46 \\
\hline RDW, \% & $13.7[13.5-14.9]$ & $13.5[12.7-14.2]$ & 0.04 \\
\hline Hb, gr/dL & $12.1[11.6-12.5]$ & $12.5[12.0-13.3]$ & 0.22 \\
\hline HCT, \% & $37.5[36.7-39.1]$ & $38.5[36.6-40.5]$ & 0.62 \\
\hline CRP, mg/dL & $3.3[1.6-6.7]$ & $3.6[1.6-5.7]$ & 0.85 \\
\hline ESR, mm/h & $43[38-55]$ & $41[34-52]$ & 0.50 \\
\hline Prednisone dose, mg/day & $15.0[15.0-15.0]$ & $15.0[15.0-15.0]$ & 0.98 \\
\hline MCV, fL & $88.7[86.5-89.3]$ & $88.5[84.4-91.7]$ & 0.91 \\
\hline MCH, pg & $28.5[27.5-29.3]$ & $28.7[27.8-30.3]$ & 0.46 \\
\hline
\end{tabular}

Continuous variables are presented as median [Interquartile range]. Categorical variables are shown as N., \%. Comparison was performed by Pearson's $\chi 2$ or Mann-Whitney test, as appropriate. Abbreviations: F, Females; M, Males; RDW, red cell distribution width; $\mathrm{Hb}$, haemoglobin; $\mathrm{HCT}$, haematocrit; CRP, C-reactive protein; ESR, erythrocyte sedimentation rate; MCV, mean corpuscular volume; $\mathrm{MCH}$, mean concentration of haemoglobin.

reports the general characteristics differences between relapsers and non-relapsers. As shown in this table, the only clinical variable which differed between groups at diagnosis was RDW ( $\mathrm{p}=0.04)$, while ESR and CRP were similar.

We then divided our population according to the median value of RDW. Out of the 9 relapsers, 8 had a baseline RDW value higher than $13.5 \% \quad\left(\chi^{2}=6.84, \mathrm{p}<0.009\right)$. Moreover, those with a value higher than $13.5 \%$ showed a significantly different relapse-free survival (Log-Rank test, $\mathrm{p}<0.03$ ) compared with those with a lower RDW, as demonstrated by the survival curves shown in Figure 1.

Interestingly, in a multiple regression analysis, including RDW, gender, age,

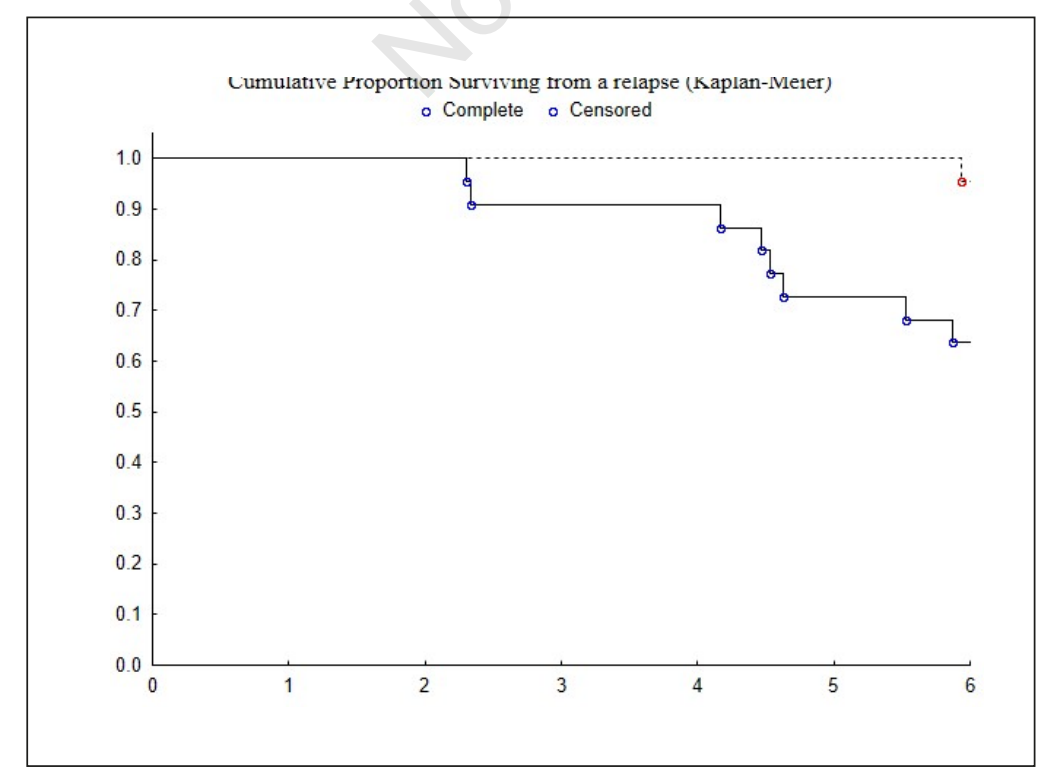

Figure 1 - Kaplan-Meier curves for relapse free-survival in patients with higher and lower RDW\%. The figure displays the Kaplan-Meier curves for relapse free-survival according to RDW\% at baseline. The continuous line represents patients with $\mathrm{RDW} \% \geq 13.5$, the dotted line of those $<13.5$. The difference is significant (Log-Rank Test, 2.21, p<0.03). 
dose of prednisone, CRP, ESR, $\mathrm{Hb}$ and $\mathrm{MCV}, \mathrm{RDW}$ was the only predictor of relapse $(p=0.01)$. This evidence was further confirmed by a stepwise logistic analysis: RDW ( $\mathrm{p}=0.01)$ predicted the risk of relapse at 6 months, while age $(\mathrm{p}=0.11)$, gender $(\mathrm{p}=0.54), \operatorname{CRP}(\mathrm{p}=0.62), \operatorname{ESR}(\mathrm{p}=0.19), \mathrm{Hb}$ $(\mathrm{p}=0.87), \operatorname{MCV}(\mathrm{p}=0.30)$ and prednisone dose $(\mathrm{p}=0.11)$ did not fit into the model.

\section{DISCUSSION AND CONCLUSIONS}

PMR is a common inflammatory condition in elderly people, which has a generally benign clinical course, with a good response to steroid treatment; however, there is a significant number of subjects who experience disease relapse during steroid tapering (10). Moreover, it might be associated to an underlying malignancy (14).

A better stratification of the risk of relapse might contribute to spare steroids and prevent side effects related to chronic glucocorticoids exposure. Unfortunately, useful predictive prognostic factors in this context do not exist: age, gender, disease activity and inflammatory marker levels have been investigated in different studies, with conflicting results (15). More recently, imaging studies, such as magnetic resonance of the shoulders, have been proposed to identify subsets of patients at higher risk or relapse, although their clinical value is not yet confirmed (16). Therefore, we performed this study, which aimed to investigate retrospectively the potential prognostic role of RDW. The rationale of our study relies on the fact that RDW is a biomarker of general health which gained visibility over the last few years in different clinical settings. Our results are in line with the current literature, since we reported that a larger RDW is a promising biomarker of early relapse in patients affected by PMR. In fact, according to our data, early relapsers show a larger RDW than non-relapsers. Moreover, the patients with a baseline RDW in the higher half of the distribution showed a significantly shorter relapse-free survival.

The mechanisms underlying the association we observed are unclear. One might speculate that RDW probably works as a surrogate inflammatory biomarker and, therefore, inflammation may be the pathogenetic factor causing anysocytosis in patients with a more aggressive and less steroid-responsive phenotype. This data is in line with a recent paper published by our group, according to which RDW outperformed ESR and CRP as prognostic biomarker in RA (10). It is interesting to note that the present study confirms this observation also in the setting of PMR, suggesting that RDW may be a more reliable marker of response to treatment than the classical inflammatory biomarkers. This observation was confirmed, when we built a logistic regression model, according to which RDW is the only predictor of early PMR relapse. It is possible to postulate that, while CRP and ESR reflect well the acute phase of inflammation, they are less efficient in the prediction of long-term outcomes. Conversely, RDW might be a better marker of the concomitant presence of disease-related organ damage. In fact, the inflammatory state may lead to nutritional deficiency and/or release of free radicals which in turn have a detrimental impact on bone marrow activity. However, this is just a speculative hypothesis. Indeed, if the baseline RDW seems to be a reliable prognostic biomarker, the CRP decay after treatment initiation has been recently described as a favorable prognostic marker. In fact, the normalization of the CRP level after one month of treatment is predictive of glucocorticoid free remission at 30 months (17). Accordingly, lower CRP levels at 6 months are predictive of remission at 12 months (18).

When we look at RDW, we should always keep in mind that it increases physiologically with age, in both genders (12). This is the reason why we decided to limit our analysis to patients younger than 90 . Therefore, we suggest to consider this limitation, when evaluating the contribution of RDW to treatment response prediction.

Our study has several limitations: firstly, the study population is relatively small. Moreover, the retrospective design of the study has intrinsic limitations, which, for 
example, did not allow to better evaluate PMR disease activity and correct for further potential confounders. In particular, RDW is influenced by many human diseases. Its increase has been associated with malignancies and inflammatory conditions, often correlating with disease activity. This makes it really difficult to correct for this crucial confounder, especially considering the small sample size.

However, it is relevant to underline the novelty of our findings, which makes them worthy of further investigation in a longitudinal study.

In conclusion, RDW is a widely available potentially useful biomarker in the management of PMR and in risk stratification for early relapse, which might allow us to tailor the treatment on an individual basis.

\section{Conflict of interests}

The authors declare no potential conflict of interests.

\section{REFERENCES}

1. Hu ZD, Lippi G, Montagnana M. Diagnostic and prognostic value of red blood cell distribution width in sepsis: A narrative review. Clin Biochem. 2020; 77: 1-6.

2. Tonelli M, Sacks F, Arnold M, et al., for the Cholesterol and Recurrent Events (CARE) Trial Investigators. Relation Between Red Blood Cell Distribution Width and Cardiovascular Event Rate in People With Coronary Disease. Circulation. 2008; 117: 163-168.

3. Patel KV, Ferrucci L, Ershler WB, et al. Red blood cell distribution width and the risk of death in middle-aged and older adults. Arch Intern Med. 2009; 169: 515-523.

4. Zorlu A, Bektasoglu G, Guven FM, et al. Usefulness of admission red cell distribution width as a predictor of early mortality in patients with acute pulmonary embolism. Am J Cardiol. 2012; 109: 128-134.

5. Zou XL, Lin XJ, Ni X, et al. Baseline red blood cell distribution width correlates with disease activity and therapeutic outcomes in patients with systemic lupus erythematosus, irrespective of anemia status. Clin Lab. 2016; 62: 1841-1850.

6. Hu ZD, Sun Y, Guo J, et al. Red blood cell distribution width and neutrophil/lymphocyte ratio are positively correlated with disease activity in primary Sjögren's syndrome. Clin Biochem. 2014; 47: 287-290.

7. Yunchun L, Yue W, Jun FZ, et al. Clinical significance of red blood cell distribution width and inflammatory factors for the disease activity in rheumatoid arthritis. Clin Lab. 2016; 62: 2327-2331.

8. Bellan M, Giubertoni A, Piccinino C, et al. Red Cell Distribution Width and Platelet Count as Biomarkers of Pulmonary Arterial Hypertension in Patients with Connective Tissue Disorders. Dis Markers. 2019; 2019: 4981982.

9. Bellan M, Soddu D, Zecca E, et al. Association between red cell distribution width and response to methotrexate in rheumatoid arthritis. Reumatismo. 2020; 72: 16-20.

10. Muratore F, Pazzola G, Pipitone N, Salvarani C. Recent advances in the diagnosis and treatment of polymyalgia rheumatica. Expert Rev Clin Immunol. 2016; 12: 1037-1045.

11. Dasgupta B, Cimmino MA, Maradit-Kremers H, et al. 2012 provisional classification criteria for polymyalgia rheumatica: a European League Against Rheumatism/American College of Rheumatology collaborative initiative. Ann Rheum Dis. 2012; 71: 484-492.

12. Hoffmann JJ, Nabbe KC, van den Broek NM. Effect of age and gender on reference intervals of red blood cell distribution width (RDW) and mean red cell volume (MCV). Clin Chem Lab Med. 2015; 53: 2015-2019.

13. Dejaco C, Singh YP, Perel P, et al. Recommendations for the management of polymyalgia rheumatica: a European League Against Rheumatism/American College of Rheumatology collaborative initiative. Ann Rheum Dis. 2015; 74: 1799-1807.

14. Bellan M, Boggio E, Sola D, Gibbin A, et al. Association between rheumatic diseases and cancer: results from a clinical practice cohort study. Intern Emerg Med. 2017; 12: 621-627.

15. Dejaco C, Singh YP, Perel P, et al. Current evidence for therapeutic interventions and prognostic factors in polymyalgia rheumatica: a systematic literature review informing the 2015 European League Against Rheumatism/ American College of Rheumatology recommendations for the management of polymyalgia rheumatica. Ann Rheum Dis. 2015; 74 : 1808-1817.

16. Nakamura H, Kamada K, Tarumi M, et al. Gadolinium-enhanced magnetic resonance imaging in shoulders contributes accurate diagnosis and predicting recurrence to patients with polymyalgia rheumatica. Clin Exp Rheumatol. 2020 Mar 28 [Epub ahead of print].

17. Hattori K, Hirano Y, Kojima T. Predictors of glucocorticoid-free remission in patients with polymyalgia rheumatica treated with prednisolone. Int $\mathrm{J}$ Rheum Dis. 2020; 23: 1581-1586.

18. Birra D, Bosello SL, Peluso G, Zoli A. Sixthmonth remission as a predictor for twelvemonth remission in polymyalgia rheumatica. Clin Exp Rheumatol. 2020; 38: 436-441. 